\title{
VALIDEZ DIAGNÓSTICA DE LA TAC HELICOIDAL FRENTE A LA ECOGRAFÍA EN EL CÓLICO RENOURETERAL
}

\author{
Diego Rengifo Abbad, Gil Rodríguez Caravaca, Laura Barreales Tolosa, Mํa Concepción Villar \\ del Campo, José Martel Villagrán y Miguel Angel Trapero García.
}

Servicio de Urología. Hospital Universitario Fundación Alcorcón. Alcorcón. Madrid. España.

Este trabajo fue financiado por el proyecto FIS 0110498

\begin{abstract}
Resumen.- OBJETIVO: La TAC helicoidal (TACh) sin contraste se evalúa como técnica diagnóstica en el cólico renoureteral. Permite un diagnóstico rápido de obstrucción ureteral y de otras patologías de sintomatología similar. Este estudio pretende evaluar la validez de la TACh frente a la ecografía en el diagnóstico de estos pacientes y plantear su inclusión, como una prueba más, en el algoritmo diagnóstico de esta patología.

MÉTODOS: Estudio de evaluación de pruebas diagnósticas realizado entre enero de 2004 y diciembre de 2006 en pacientes valorados por cólico renoureteral no resuelto con atención estándar, estudiándolos de
\end{abstract}

\section{CORRESPONDENCIA}

Diego Rengifo Abbad

Unidad de Urología

Hospital Universitario Puerta de Hierro

Majadahonda.

Manuel de Falla, 1 .

28222 Majadahonda. Madrid. (España).

drengifo22@yahoo.es

Aceptado para publicar: 20 de abril 2009 forma ciega mediante ecografía y TACh y siguiéndolos hasta confirmar el diagnóstico con un "patrón oro". Las variables cualitativas se resumen con su distribución de frecuencias e IC $95 \%$ y las cuantitativas con medidas de posición y dispersión. Se utilizó el test de $\chi^{2}$ para comparar variables cualitativas. Se calcularon los índices de validez diagnóstica con sus IC95\% y se compararon con la prueba de McNemar.

RESULTADOS: Se estudió a 124 pacientes, presentando litiasis el 59,7\% y otros diagnósticos el 40,3\%. Las 92 ecografías efectuadas diagnosticaron un $22,8 \%$ de litiasis frente al 59,7\% obtenido con 124 TACh. Los indices de validez para la TACh fueron superiores al $95 \%$, con una sensibilidad y valor predictivo positivo (VPP) de 100\%. La ecografía presentó una especificidad y un VPP de $100 \%$ pero sensibilidad y valor predictivo negativo de 29,6\% ( $p<0,05)$.

CONCLUSIÓN: La TACh puede ser considerada una prueba más a utilizar en el diagnóstico del cólico renoureteral.

Palabras clave: Ecografía. Cólico nefrítico. Validez diagnóstica. TAC helicoidal.

Summary.- OBJECTIVES: Helical CT without contrast is currently being evaluated for the diagnosis of renoureteral colic. Ureteral obstruction and other pathologies with similar symptoms can be rapidly identified. This study intends to evaluate the validity of CTh for these patients and to compare it with the ultrasound technique in order to consider the CTh as an efficient diagnostic alternative for these pathologies.

METHODS: A diagnostic evaluation study was carried out between January 2004 and December 2006 in 
patients with persistent renoureteral colic after standard care. They were blindly evaluated by ultrasound and CTh, maintaining the follow up until the "gold standard" confirmation. Qualitative variables were described by frequency and $95 \% \mathrm{Cl}$ and quantitative variables by position and dispersion measures. The $\chi^{2}$ test was used to compare qualitative variables. Validity indicators and 95\% Cl were calculated and compared with McNemar test.

RESULTS: 124 patients were studied. Lithiasis and the other diagnosis had frequencies of $59.7 \%$ and $40.3 \%$ respectively. The percentage of lithiasis obtained by ultrasound (92) was $22.8 \%$ and by CTh (124) was $59.7 \%$.

Validity indicators for CTh were superior to 95\%, with $100 \%$ sensitivity and positive predictive value. Ultrasound specificity and positive predictive value were 100\%, but sensitivity was only $29.6 \%(p<0.05)$.

CONCLUSION: CTh can be considered a good alternative diagnostic technique in the renoureteral colic management.

Keywords: Ureteral calculi. Helical TC. Echography. Reproducibility of results.

\section{INTRODUCCIÓN}

La presencia de dolor cólico en flanco representa un problema clínico muy común y complejo. En la mayoría de los casos este dolor se produce como consecuencia de una obstrucción aguda ocasionada por una litiasis ureteral, pero existen otras patologías que provocan cuadros clínicos similares. Por todo ello y debido a que la litiasis es la causa más frecuente, se debe descartar su presencia ante un paciente con dolor cólico en flanco (1). Las técnicas de imagen son las que complementan, junto con las analíticas, el diagnóstico semiológico (2).

La TAC helicoidal (TACh) sin contraste para la evaluación de pacientes con dolor cólico y sospecha de obstrucción ureteral por litiasis, fue descrita por primera vez en 1994 (3) y publicada por Smith en 1995 (4). Desde entonces se viene aceptando como la técnica de imagen que sustituye a las previamente descritas (5-7) (radiografía simple, ecografía y urografía intravenosa) en el diagnóstico de estos pacientes, debido a que permite un diagnóstico rápido y preciso de la presencia de obstrucción ureteral y de otras patologías de sintomatología similar (8). Entre sus ventajas destaca el no necesitar contraste intravenoso ni preparación intestinal previa, así como el hecho de permitir la visualización de todos los tipos de litiasis y aportar información precisa acerca de la localización y el tamaño de las mismas. Sin embargo, a pesar de las ventajas de la TACh, su uso no está aún generalizado en el ámbito sanitario español. Este estudio pretende, por tanto, evaluar la validez de la TACh frente a la ecografía en el diagnóstico del cólico renoureteral con objeto de, en caso de obtener resultados óptimos, integrarla como una prueba más en el algoritmo diagnóstico de esta patología.

\section{MATERIAL Y MÉTODOS}

\section{Objetivo}

Evaluar la validez diagnóstica de la TACh sin contraste y de la ecografía en el estudio del cólico renoureteral complicado.

\section{Diseño del estudio} diagnósticas.

Estudio descriptivo de evaluación de pruebas

\section{Ámbito y periodo de estudio}

Población de referencia de la Fundación Hospital Alcorcón (FHA, Comunidad de Madrid), que es de unos 250.000 habitantes. El periodo estudiado estaba comprendido entre el 1 de enero de 2004 y el 31 de diciembre de 2006 como límite del seguimiento clínico para la determinación de la causa definitiva de la patología.

\section{Criterios de inclusión}

Cualquier paciente perteneciente a la población de referencia de la FHA que hubiera sido valorado de forma urgente en Atención Primaria, en el Servicio de Urgencias de nuestro centro, en las consultas de Medicina Interna o en las consultas de Urología por un cuadro de dolor cólico renoureteral no resuelto con atención estándar, es decir, con valoración clínica completa y tratamiento conservador con analgésicos, antiinflamatorios y diuresis forzada.

\section{Criterios de exclusión}

Se excluyó a los pacientes que no cumplían los criterios de inclusión, a las mujeres con sospecha o confirmación de embarazo, a los sujetos con insuficiencia renal (creatinina $>2,5 \mathrm{mg} / \mathrm{dl}$ ) y a los que no firmaron el consentimiento informado.

\section{Plan de trabajo}

Los pacientes que cumplían los criterios de inclusión fueron sometidos, previo consentimiento informado, al estudio de imagen con ecografía y TACh $y$, en la mayoría de los casos, también fueron estudiados mediante análisis de orina y sangre. La evaluación de las pruebas diagnósticas se realizó con enmascaramiento, de forma que los radiólogos eva- 
luadores desconocían el resultado de las diferentes pruebas realizadas al mismo paciente. La ecografía era realizada a tiempo real utilizando el ecógrafo General Electric Logia 400 del departamento de imagen, con sonda de $3,5 \mathrm{MHz}$, y en algunos casos el B\&K 2001 Leopard de la Unidad de Urología. Las pruebas de TACh se realizaron con un modelo ProSpeed SX Advantage (General Electric) y utilizando un protocolo estándar sin administración de contraste.

El seguimiento de los participantes se mantenía hasta ser posible la determinación del origen del cólico renoureteral, confirmándolo mediante pruebas "patrón oro", como el estudio anatomopatológico de las lesiones en caso de patología tumoral, la expulsión espontánea o quirúrgica de las piedras en caso de litiasis o el diagnóstico de cualquier otra patología con el patrón oro correspondiente. Todos los pacientes incluidos en el estudio fueron seguidos

TABLA I. CARACTERISTICAS DE LOS PACIENTES CON DIAGNÓSTICO DE LITIASIS (N=74).

\begin{tabular}{|c|c|}
\hline \multirow[b]{2}{*}{ Localización (TAC) } & n (\%) \\
\hline & \\
\hline Renal & $4(5,4)$ \\
\hline Uréter proximal & $14(18,9)$ \\
\hline Uréter medio & $11(14,9)$ \\
\hline Uréter distal & $21(28,4)$ \\
\hline Unión ureterovesical & $23(31,1)$ \\
\hline Vejiga & $1(1,4)$ \\
\hline \multicolumn{2}{|l|}{ Tamaño medio } \\
\hline$>5 \mathrm{~mm}$ & $35(47,3)$ \\
\hline$<5 \mathrm{~mm}$ & $39(52,7)$ \\
\hline Densidad* & $460,3(231,7)$ \\
\hline \multicolumn{2}{|l|}{ Resolución } \\
\hline Expulsión espontánea & $41(55,4)$ \\
\hline Derivación JJ & $1(1,4)$ \\
\hline Ureteroscopia & $12(16,2)$ \\
\hline Litotricia & $13(17,6)$ \\
\hline Alcalinización & $5(6,8)$ \\
\hline LEOC* + URS* & $2(2,7)$ \\
\hline \multicolumn{2}{|l|}{ Composición } \\
\hline Oxalato cálcico & $36(48,6)$ \\
\hline Fosfato amónico mg & $1(1,4)$ \\
\hline Ácido úrico & $4(54,1)$ \\
\hline Mixto & $2(2,7)$ \\
\hline No recogido & $31(41,9)$ \\
\hline
\end{tabular}

clínicamente hasta que se tuvo un diagnóstico patrón oro de cada paciente.

\section{Las variables estudiadas fueron}

Edad, sexo, antecedentes personales y familiares relacionados con patología litiásica, fecha de presentación de la clínica, signos y síntomas de presentación, resultados del análisis de orina y resultados de TACh y ecografía. Los resultados de la TACh y de la ecografía se codificaron como presencia o ausencia de litiasis. La TACh permitió también evaluar la localización topográfica de la litiasis (renal, uréter proximal, uréter medio, uréter distal, unión ureterovesical, vejiga) y el diámetro en milímetros recodificado ( $\leqslant 5 \mathrm{~mm} ; \geq 5 \mathrm{~mm}$ ). Se evaluó también la densidad de las imágenes de litiasis en Unidades Hounsfield densitométricas (UH). Finalmente se registró la forma de resolución del cuadro clínico, el tipo de cálculo en caso de litiasis y el resultado del patrón oro de cada paciente (diagnóstico definitivo).

\section{Análisis estadístico}

Se efectuó una estimación del tamaño muestral para un nivel de confianza del $95 \%$ (error $\alpha$ de $0,05)$, una precisión del $5 \%$, un error $\beta$ del $20 \%$, una diferencia entre el peor índice de validez diagnóstica de las pruebas del $15 \%$, y asumiendo un $10 \%$ de pérdidas. Las variables cualitativas se resumen con su distribución de frecuencias e intervalo de confianza al $95 \%$ y las cuantitativas con medidas de posición (media o mediana) y de dispersión (desviación estándar o rango intercuartílico). Se utilizó el test de $\chi^{2}$ para la comparación de variables cualitativas. Se calcularon los índices de validez diagnóstica (sensibilidad, especificidad, valor global y valores predictivos) con sus intervalos de confianza al $95 \%$. Los índices de validez entre ambas pruebas se compararon con la prueba de McNemar para datos apareados. El nivel de significación en todos los contrastes de hipótesis fue de 0,05. El programa utilizado para el análisis fue SPSS 14. Así se estimó necesario estudiar 126 pacientes.

\section{RESULTADOS}

Se seleccionaron 159 pacientes diagnosticados de cólico renoureteral complicado, excluyendo con posterioridad a 35 de ellos por no cumplir los criterios de inclusión, por lo que finalmente se disponía de una muestra de 124 pacientes evaluables. El 58\% de los sujetos eran hombres y el $42 \%$ mujeres. De los 124 pacientes presentaban el diagnóstico de litiasis $74(59,7 \%)$ y $50(40,3 \%)$ otros diagnósticos diferenciales. La edad media en el grupo de litiasis fue de 48,5 años (rango: $24-81$ ) y de 38,5 años (rango: $24-75$ ) en el grupo de diagnósticos diferenciales. 


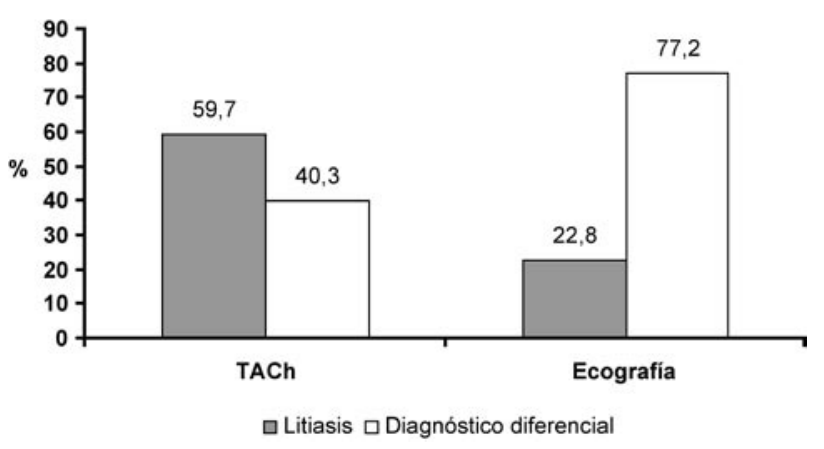

FIGURA 1. Distribución de frecuencias de los diagnósticos informados por TACh y ecografía.

El $65,8 \%$ de los sujetos con litiasis presentaban alteraciones en el sedimento de orina. En la Tabla I se describen las características estudiadas en los pacientes con litiasis. Dentro de las patologías no litiásicas, destacaron las relacionadas con el aparato urinario, especialmente la pielonefritis, presente en el $16 \%$ de los sujetos. En el $36 \%$ de los sujetos sin litiasis no se encontraron hallazgos patológicos reseñables.

Se realizaron 124 TACh y 92 ecografías en los pacientes con cólico renal complicado. Las 92 ecografías permitieron informar 21 litiasis $(22,8 \%)$ y 71 diagnósticos diferenciales $(77,2 \%)$. Con la TACh se detectaron 74 casos de litiasis $(59,7 \%)$ y 50 diagnósticos diferenciales $(40,3 \%)$. El porcentaje de diagnósticos de litiasis fue significativamente superior al realizar TACh $(p<0,0001)$, del mismo modo que la ecografía permitió detectar un porcentaje sig- nificativamente mayor de diagnósticos diferenciales $(p<0,0001)$.

La TACh mostró una sensibilidad y un valor predictivo negativo del 100\%. El resto de los índices de validez diagnóstica fueron superiores al $95 \%$, con unos intervalos de confianza muy precisos. La ecografía presentó una especificidad y un valor predictivo positivo del $100 \%$ pero una sensibilidad y valor predictivo negativo bajos $(29,6 \%$ en ambos casos) y menor precisión en los intervalos de confianza. En la Tabla Il se resumen los índices de validez diagnóstica de ambas pruebas con sus intervalos de confianza al $95 \%$.

El tipo de resolución clínica más frecuente, para los pacientes con litiasis, fue la expulsión espontánea $(55,4 \%)$. Un elevado porcentaje de las litiasis con un tamaño $\geq 5 \mathrm{~mm}$ precisó tratamiento resolutivo $(76,5 \%)$.

\section{DISCUSIÓN}

Es importante realizar un diagnóstico clínico con una alta validez, especialmente en aquellas enfermedades que pueden suponer una demanda excesiva de consultas y pruebas y en aquellas enfermedades graves.

La ecografía permite visualizar las litiasis localizadas en riñón, pelvis renal y algunas situadas en las proximidades de la unión ureterovesical. La sensibilidad de la ecografía para todas las localizaciones en esta serie fue baja $(29,7 \%)$, con una elevada predisposición a obtener falsos negativos. Si se

TABLA II. ÍNDICES DE VALIDEZ DIAGNÓSTICA DE TACH SIN CONTRASTE Y ECOGRAFÍA.

\begin{tabular}{|l|cc|cc|c|}
\hline & \multicolumn{2}{|c|}{ TACh SIN CONTRASTE } & \multicolumn{2}{c|}{ ECOGRAFÍA } & \\
& Valor (\%) & IC 95\% & Valor (\%) & IC 95\% & p \\
\hline Sensibilidad & 100,0 & $100,0-100,0$ & 29,6 & $18,9-40,2$ & $<0,05$ \\
Especificidad & 95,8 & $90,2-100,0$ & 100,0 & $100,0-100,0$ & $>0,05$ \\
VPP* & 97,4 & $93,8-100,0$ & 100,0 & $100,0-100,0$ & $>0,05$ \\
VPN** & 100,0 & $93,4-100,0$ & 29,6 & $18,9-40,2$ & $<0,05$ \\
Valor global & 98,4 & $96,0-100,0$ & 45,7 & $35,5-55,8$ & $<0,05$ \\
\hline
\end{tabular}

* Valor predictivo positivo

** Valor predictivo negativo 
tiene en cuenta que el cólico se produce generalmente cuando la litiasis se aloja en el uréter y produce obstrucción carecerían de interés las ubicadas a nivel renal, excepto las asociadas a litiasis ureteral, por lo que la sensibilidad de la ecografía sería aún menor que la obtenida, aproximadamente de un $20 \%$. La especificidad obtenida fue máxima $(100 \%)$ por lo que la posibilidad de obtener falsos positivos es extremadamente baja.

Varios autores $(9,10)$ han utilizado la radiografía simple y la ecografía para evaluar a los pacientes con sospecha de litiasis ureteral pero, la presencia de una calcificación en la radiografía no asegura que sea la responsable de la dilatación del sistema colector encontrado en la ecografía. En un estudio reciente, Catalano (11) comparó los resultados obtenidos en pacientes con cólico renal a los que se les realizó, entre otras pruebas, ecografía y radiografía simple (48 pacientes) y TACh (48 pacientes), encontrando una mayor precisión en el grupo de TACh aunque la radiografía y la ecografía obtuvieron resultados similares. Los autores abogaron por el uso generalizado de estas dos pruebas, reservando la TACh para uropatías obstructivas. Sin embargo, esta aseveración no es compartida por todos los autores, ya que la interpretación de la ecografía es subjetiva y los índices de validez obtenidos para ambas pruebas no fueron muy altos. Por otra parte, la radiografía simple de abdomen no permite visualizar litiasis ureterales en un número importante de casos y debe ser utilizada teniendo en cuenta sus limitaciones (12).

La urografía intravenosa (13) precisa de preparación intestinal previa y del uso de contraste. Evidencia la presencia de obstrucción ureteral por lo que, en caso de litiasis de pequeño tamaño, presenta los mismos inconvenientes que la radiografía simple y, por otra parte, no aporta información sobre patologías extraurinarias que producen cuadros clínicos similares (14). Aunque, como ventaja con respecto al resto de las pruebas, permite conocer la funcionalidad renal, esta información no altera el manejo del paciente en la mayoría de las ocasiones. Los estudios de validez de la urografía intravenosa demuestran unos índices diagnósticos inferiores a la TACh (15).

Una de las principales ventajas de la TACh es la visualización de la localización y tamaño de las litiasis (16-18), los dos factores más importantes en el manejo del paciente debido, en parte, a su relación con la posibilidad de expulsión espontánea. No hay tanta ventaja en cuanto a la precisión para diferenciar el grado de obstrucción entre alto y parcial (19).
La validez diagnóstica obtenida en esta serie para la TACh es casi perfecta, con un valor global próximo al $100 \%$, presentando finalmente mayor validez que la ecografía en el diagnóstico del cólico renoureteral, coincidiendo con estudios recientemente realizados (6).

Las principales limitaciones del estudio intentaron controlarse. Así por ejemplo, el "sesgo de sospecha diagnóstica" se controló mediante la realización ciega de las dos pruebas. Creemos que el "sesgo de selección" de la muestra ha sido controlado y no debe alterar los resultados, puesto que los pacientes con criterios de inclusión tenían como hospital de referencia la FHA y eran derivados para valoración y tratamiento a las consultas externas de medicina interna, a las consultas externas de urología o al servicio de urgencias, donde se puso en marcha todo el estudio. Las pérdidas por demora, búsqueda de atención en otros centros y otras eventualidades se consideraron al corregir, con un porcentaje de pérdidas, la estimación del tamaño muestral.

\section{CONCLUSIONES}

Teniendo en cuenta estos resultados y las ventajas que aporta esta técnica, comentadas anteriormente, se puede concluir que la TACh puede ser considerada una prueba más a utilizar en el diagnóstico del cólico renoureteral, con el valor añadido de poder modificar el manejo del paciente, al informar sobre la localización y el tamaño de las litiasis. Aún así, la disponibilidad de la prueba, la dosis de radiación y el coste han de tenerse también en cuenta $(20,21)$.

\section{BIBLIOGRAFÍA y LECTURAS RECOMENDADAS (*lectura de interés $y^{* *}$ lectura fundamental)}

1. Teichman JM. Clinical practice. Acute renal colic from ureteral calculus. N Engl Med, 2004; 350(7): 684-93.

2. Bishop NL. The influence of emergency urography and hematuria in the diagnosis of ureteric colic. Clin Radiol, 1980; 31: 605-10.

**3. Essenmacher KR, Smith RC, Rosenfield AT. Comparison of unhenhanced CT to excretory urography in evaluating patients with flank pain. Annual meeting of the Society of Uroradiology, Laguna Nigel, CA, Jan 1994.

**4. Smith RC, Rosenfield AT, Choe Kal . Acute flank pain: comparison of unhenhanced-enhanced CT and intravenous urography. Radiol, 1995; 194: 789-94. 
*5. Ahmad NA, Ather MH, Rees J. Unenhanced helical computed tomography in the evaluation of acute flank pain. Int J Urol, 2003; 10(6): 287-92.

6. Ripolles T, Errando J, Agramunt M, Martínez MJ. Ureteral colic : US vs CT. Abdom Imaging, 2004; 29(2): 263-6.

7. De Souza LR, Goldman SM, Faintuch S, Faria JF, Bekhor D, Tiferes DA et al. Comparison between ultrasound and noncontrast helical computed tomography for identification of acute ureterolithiasis in a teaching hospital stting. Sao Paulo Med J, 2007; 125(2): 102-7.

8. Iturralde Codina A, Muñoz YI. Apendicitis aguda versus cólico nefrítico derecho. Arch Esp Urol, 2006: 59: 3 .

9. Laing FC, Jeffrey RB, Wing BW. Ultrasound vs excretory urography in evaluating acute flank pain. Radiol, 1985; 154: 613-6.

10. Hill MC, Rich JI, Maridat JG, Finder CA. Sonography vs excretory urography in acute flank pain. AJR, 1985; 144: 1235-8.

*11. Catalano O, Nunziata A, Altei F, Siani A. Suspected ureteral colic: primary helical CT versus selective helical CT after unenhanced radiography and sonography. AJR, 2002; 178(2): 379-87.

12. Moyano Calvo JL, Huesa Martínez I, Cruz Navarro N, Leal Arenas J, León Dueñas E, Morales Lopez A, et al. Litiasis urinaria secundaria a Indinavir en enfermo HIV positivo. Arch Esp Urol, 2001; 54: 10.

13. Osborne ED, Sutherland CG, Scholl AJ, Rowntree LG. Roentgenography of urinary tract during excretion of sodium iodide. J.A.M.A. 1923; 80: 368.

14. Soto Villalba J, Flores Ortiz J, Gutierrez Somoscarrera JA, Flores Ginéz J. Cólico nefrítico por fibrosis periureteral de metastásis de carcinoma lobulillar de mama. Arch Esp Urol, 2001; 54: 2.

15. Jia-Hwia W, Shu-Huei S, Shan-Su H, Cheng-Yen $\mathrm{C}$, Prospective comparison of unenhanced spiral computed tomography and intravenous urography in the evaluation of acute renal colic. J Chin Med Assoc, 2008; 71(1): 30-6.

16. Olcott EW, Sommer FG, Napel S. Accuracy of detection and measurement of renal calculi: in-vitro comparison of three dimensional spiral CT, radiography and nephrotomography. Radiol, 1997; 204: 19-25.

*17. Fielding JR, Fox LA, Heller H. Spiral CT in the evaluation of acute flank pain: overall accuracy and feature análisis. J Comput Assist Tomogr, 1997; 21: 635-638.

*18. Neitlich J, Hodges I, Barish M. The accuracy of helical CT in determining renal stone size and volume. Annual meeting of the Society of Uroradiology, Santa Fe, NM, June. 1997.

19. Bird VG, Gómez-Marín O, Leveillee RJ, Sfakianakis GN, Rivas LA, Amendola MA. A comparison of unenhanced helical computerized tomography findings and renal obstruction determined by furosemide 99 mtechnetium mercaptoacetyltriglycine diuretic scintirenography for patients with acute renal colic. Journ Urol, 2002; 167: 1597603.

20. Strohmaier WL, Bartunek R. Diagnostic imaging: the end of intravenous urography?. Urol A, 2008; 47(5): 556-62.

21. Kalra MK, Maher MM, D’Souza RV, Rizzo S, Halpern EF, Blake MA et al. Detection of urinary tract stones at low-radiation-dose $\mathrm{CT}$ with $\mathrm{Z}$-axis automatic tube current modulation: phanton and clinical studies. Radiol, 2005; 235(2): 523-9. 